Supporting Information:

\title{
Viologen-Based Redox-Active Ionic Liquid Crystals Forming Columnar Phases
}

\author{
Kana Tanabe, Takuma Yasuda, Masafumi Yoshio, and Takashi Kato* \\ Department of Chemistry and Biotechnology, School of Engineering, \\ The University of Tokyo, Hongo, Bunkyo-ku, Tokyo 113-8656, Japan
}

\section{Experimental Details:}

General. ${ }^{1} \mathrm{H}$ and ${ }^{13} \mathrm{C}\left\{{ }^{1} \mathrm{H}\right\}$ NMR spectra were recorded on a JEOL JNM-LA400 spectrometer. Mass spectra were obtained with a PerSeptive Biosystems Voyager-DE STR spectrometer. Elemental analyses were carried out with a Yanaco MT-6 CHN autocorder. Differential scanning calorimetry (DSC) measurements were performed on a NETZSCH DSC204 Phoenix calorimeter at a scanning rate of $10{ }^{\circ} \mathrm{C} \mathrm{min}^{-1}$. A polarizing optical microscope Olympus BH-51 equipped with Mettler FP82 HT hot stage was used for visual observation. X-ray diffraction (XRD) patterns were obtained using a Rigaku RINT-2500 diffractmeter with a heating stage using Ni-filtered CuKa radiation. Cyclic voltammetry $(\mathrm{CV})$ was carried out in a $\mathrm{CH}_{2} \mathrm{Cl}_{2}$ solution of $\mathrm{Bu}_{4} \mathrm{NClO}_{4}(0.10 \mathrm{M})$ with Pt working and counter electrodes and an $\mathrm{Ag}^{+} / \mathrm{Ag}$ reference electrode by using an ALS CHI 600B electrochemical analyzer. In situ electrochromism studies were conducted in a three-electrode quartz cell with the same electrolyte system as CV using the potentiostat together with a JASCO V-670 spectrometer. 
X-ray Crystallographic Analysis. Single crystals of viologen 4 were obtained by slow diffusion of methanol into an acetone solution. The measurements were made on a Rigaku Mercury CCD diffractmeter with graphite monochromated MoK $\alpha$ radiation. Data were processed using the Rigaku Crystalclear software package. The structure of 4 was solved by a direct method with SIR- $97^{1}$ and refined by a full-matrix least-squares technique (SHELXL-97²). Structural parameters of non-hydrogen atoms were refined anisotropically. The crystallographic data for $\mathbf{4}$ are given in the accompanying CIF file.

Materials and Syntheses. All reagents and solvents were purchased from Aldrich, Tokyo Kasei, Kanto Chemical, or Wako, and used as received. The synthetic routes used to obtain viologens 1-4 are shown in Scheme S1. Methyl-3,4,5-trialkoxybenzoate 5a-c were prepared according to the literature. ${ }^{3}$ All reactions were performed under an Ar atmosphere in dry solvents, unless otherwise noted.
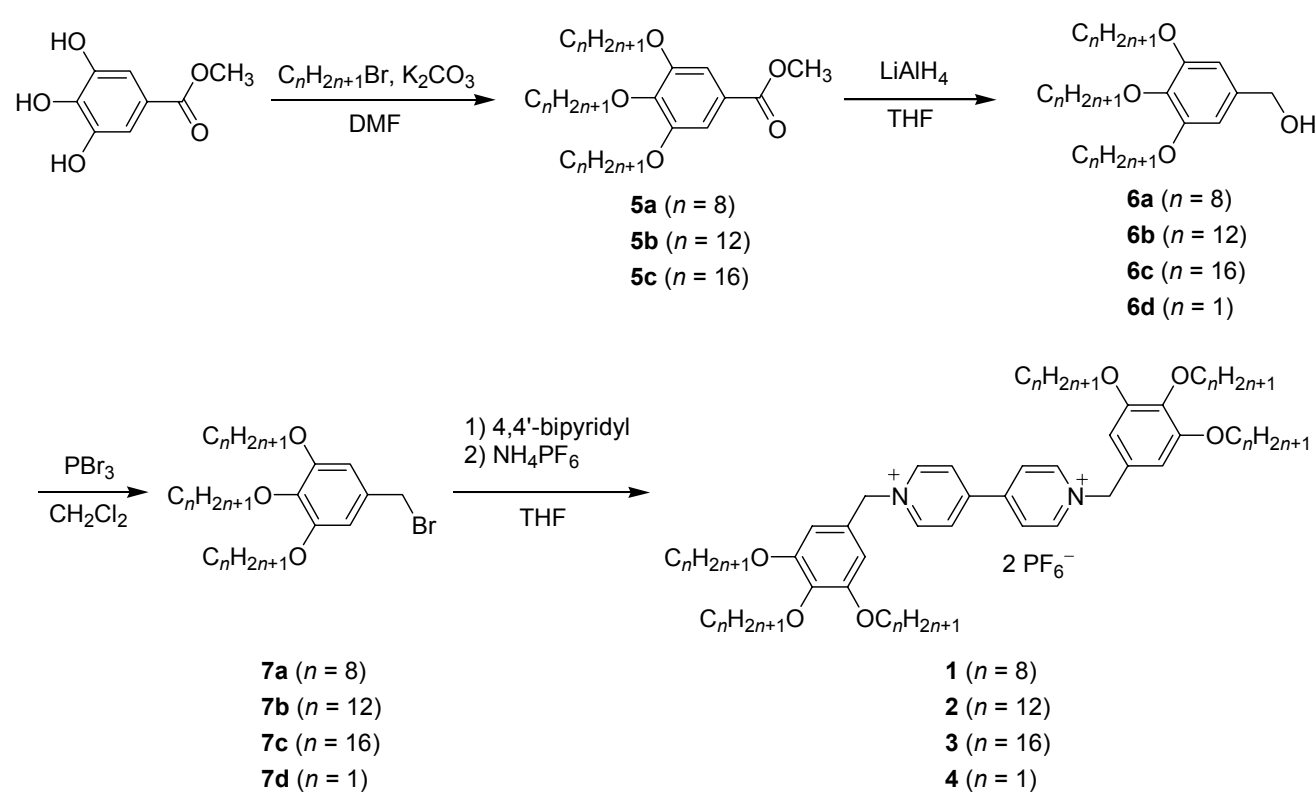

Scheme S1. Synthesis of liquid-crystalline viologens (1-3) and the model compound (4). 
3,4,5-Trioctyloxybenzyl alcohol (6a). To a stirred suspension of $\mathrm{LiAlH}_{4}(1.90 \mathrm{~g}$, $50 \mathrm{mmol})$ in dry THF $(30 \mathrm{~mL})$ was added dropwise a solution of $\mathbf{5 a}(20.8 \mathrm{~g}, 40 \mathrm{mmol})$ in THF $(50 \mathrm{~mL})$ at $0{ }^{\circ} \mathrm{C}$. After stirring for $12 \mathrm{~h}$ at room temperature, the reaction mixture was quenched with a small amount of methanol and water. The insoluble materials were filtered off, and the filtrate was concentrated under a reduced pressure. The residue was dissolved in $\mathrm{CHCl}_{3}$, washed with water and brine, and dried over anhydrous $\mathrm{Na}_{2} \mathrm{SO}_{4}$. After filtration and evaporation, the product was recrystallized from ethyl acetate to afford $\mathbf{6 a}$ as a white solid (yield $=15.5 \mathrm{~g}, 79 \%$ ). $\quad$ Mp 51-52 ${ }^{\circ} \mathrm{C}$. ${ }^{1} \mathrm{H}$ NMR $\left(400 \mathrm{MHz}, \mathrm{CDCl}_{3}\right): \delta 6.55(\mathrm{~s}, 2 \mathrm{H}), 4.58(\mathrm{~d}, J=6.0 \mathrm{~Hz}, 2 \mathrm{H}), 3.99-3.91(\mathrm{~m}$, $6 \mathrm{H}), 1.83-1.70(\mathrm{~m}, 6 \mathrm{H}), 1.50-1.20(\mathrm{~m}, 30 \mathrm{H}), 0.88(\mathrm{t}, J=6.8 \mathrm{~Hz}, 9 \mathrm{H}) . \quad{ }^{13} \mathrm{C}\left\{{ }^{1} \mathrm{H}\right\} \mathrm{NMR}$ $\left(100 \mathrm{MHz}, \mathrm{CDCl}_{3}\right): \delta 153.23,137.52,136.03,105.29,73.41,69.06,65.62,31.89,31.81$, $30.29,29.54,29.39,29.34,29.27,26.10,26.08,22.67,22.65,14.07$. MS (MALDI): $m / z 515.31\left(\right.$ calcd $\left.[M+\mathrm{Na}]^{+}=515.41\right)$.

Preparation of compounds $\mathbf{6 b}$ and $\mathbf{6 c}$ was carried out analogously, and they gave the following spectroscopic data.

3,4,5-Tridodecyloxybenzyl alcohol (6b). White solid (yield $=97 \%$ ). $\quad$ Mp $52-53{ }^{\circ} \mathrm{C} . \quad{ }^{1} \mathrm{H}$ NMR $\left(400 \mathrm{MHz}, \mathrm{CDCl}_{3}\right): \delta 6.56(\mathrm{~s}, 2 \mathrm{H}), 4.60(\mathrm{~d}, J=6.0 \mathrm{~Hz}, 2 \mathrm{H})$, 3.99-3.91 (m, 6H), 1.83-1.72 (m, 6H), 1.50-1.20 (m, 54H), $0.88(\mathrm{t}, J=6.8 \mathrm{~Hz}, 9 \mathrm{H})$. ${ }^{13} \mathrm{C}\left\{{ }^{1} \mathrm{H}\right\} \mathrm{NMR}\left(100 \mathrm{MHz}, \mathrm{CDCl}_{3}\right): \delta 153.22,137.46,136.05,105.24,73.41,69.04$, $65.59,31.91,30.30,29.75,29.72,29.69,29.65,29.63,29.61,29.45,29.40,29.36,26.12$, 26.09, 22.67, 14.10. MS (MALDI): $m / z 660.32\left(\right.$ calcd $\left.[M]^{+\cdot}=660.61\right), 683.31$ (calcd $\left.[M+\mathrm{Na}]^{+}=683.60\right)$.

3,4,5-Trihexadecyloxybenzyl alcohol (6c). White solid (yield $=99 \%$ ). $\quad \mathrm{Mp}$ 54-55 ${ }^{\circ} \mathrm{C} . \quad{ }^{1} \mathrm{H}$ NMR $\left(400 \mathrm{MHz}, \mathrm{CDCl}_{3}\right): \delta 6.53(\mathrm{~s}, 2 \mathrm{H}), 4.56(\mathrm{~d}, J=6.0 \mathrm{~Hz}, 2 \mathrm{H})$, 3.99-3.91 (m, 6H), 1.85-1.70 (m, 6H), 1.50-1.20 (m, 78H), $0.88(\mathrm{t}, J=6.8 \mathrm{~Hz}, 9 \mathrm{H})$. 
${ }^{13} \mathrm{C}\left\{{ }^{1} \mathrm{H}\right\}$ NMR $\left(100 \mathrm{MHz}, \mathrm{CDCl}_{3}\right): \delta 153.28,137.50,135.98,105.31,73.42,69.09$, $65.70,31.93,30.32,30.31,29.76,29.72,29.70,29.66,29.63,29.54,29.46,29.42,29.36$, $28.89,28.18,26.89,26.14,26.10,22.69,14.12$. MS (MALDI): $m / z 828.40$ (calcd $\left.[M]^{+\cdot}=828.79\right), 851.39\left(\right.$ calcd $\left.[M+N a]^{+}=851.78\right)$.

3,4,5-Trioctyloxybenzyl bromide (7a). To a stirred solution of $6 \mathbf{a}$ (14.8 g, 30 mmol) in $\mathrm{CH}_{2} \mathrm{Cl}_{2}(100 \mathrm{~mL})$ was added a solution of $\mathrm{PBr}_{3}(16.2 \mathrm{~g}, 60 \mathrm{mmol})$ in $\mathrm{CH}_{2} \mathrm{Cl}_{2}$ $(10 \mathrm{~mL})$ at $0{ }^{\circ} \mathrm{C}$. The mixture was allowed to react for $3 \mathrm{~h}$ at room temperature, and then poured into a large amount of water. The product was extracted with $\mathrm{CHCl}_{3}$ three times. The combined organic layers were washed with brine, and dried over anhydrous $\mathrm{Na}_{2} \mathrm{SO}_{4}$. After filtration and evaporation, the product was dried under vacuum to give $7 \mathbf{a}$ as a colorless oil (yield $=15.5 \mathrm{~g}, 93 \%$ ). The freshly prepared $7 \mathbf{a}$ was used immediately without further purification in the next reaction step. ${ }^{1} \mathrm{H}$ NMR $\left(400 \mathrm{MHz}, \mathrm{CDCl}_{3}\right): \delta 6.57(\mathrm{~s}, 2 \mathrm{H}), 4.43(\mathrm{~s}, 2 \mathrm{H}), 3.98-3.92(\mathrm{~m}, 6 \mathrm{H}), 1.83-1.69(\mathrm{~m}, 6 \mathrm{H})$, 1.50-1.20 (m, 30H), $0.89(\mathrm{t}, J=6.8 \mathrm{~Hz}, 9 \mathrm{H}) .{ }^{13} \mathrm{C}\left\{{ }^{1} \mathrm{H}\right\}$ NMR $\left(100 \mathrm{MHz}, \mathrm{CDCl}_{3}\right)$ : $\delta 153.12,138.35,132.48,107.46,73.42,69.08,34.57,31.87,31.80,30.29,29.51,29.33$, $29.26,26.05,22.66,22.64,14.06$.

Other 3,4,5-Trialkoxybenzyl bromides were prepared in a similar manner to 7a, and were used immediately without further purification in the next reaction step. Spectroscopic data of $\mathbf{7 b - d}$ are shown below.

3,4,5-Tridodecyloxybenzyl bromide (7b). White solid (yield $=98 \%$ ). $\quad{ }^{1} \mathrm{H}$ NMR $\left(400 \mathrm{MHz}, \mathrm{CDCl}_{3}\right): \delta 6.57(\mathrm{~s}, 2 \mathrm{H}), 4.43(\mathrm{~s}, 2 \mathrm{H}), 3.98-3.92(\mathrm{~m}, 6 \mathrm{H}), 1.82-1.70(\mathrm{~m}, 6 \mathrm{H})$, 1.50-1.20 (m, 54H), $0.88(\mathrm{t}, J=6.8 \mathrm{~Hz}, 9 \mathrm{H}) .{ }^{13} \mathrm{C}\left\{{ }^{1} \mathrm{H}\right\}$ NMR $\left(100 \mathrm{MHz}, \mathrm{CDCl}_{3}\right)$ : $\delta 153.11,138.27,132.49,107.39,73.43,69.04,34.60,31.91,30.29,29.73,29.68,29.64$, $29.62,29.59,29.39,29.36,29.33,26.08,26.07,22.67,14.10$.

3,4,5-Trihexadecyloxybenzyl bromide $(7 \mathrm{c})$. White solid (yield $=83 \%$ ). $\quad{ }^{1} \mathrm{H}$ 
NMR (400 MHz, $\left.\mathrm{CDCl}_{3}\right): \delta 6.57(\mathrm{~s}, 2 \mathrm{H}), 4.42(\mathrm{~s}, 2 \mathrm{H}), 3.98-3.92(\mathrm{~m}, 6 \mathrm{H}), 1.84-1.70$ $(\mathrm{m}, 6 \mathrm{H}), 1.50-1.20(\mathrm{~m}, 78 \mathrm{H}), 0.88(\mathrm{t}, J=6.8 \mathrm{~Hz}, 9 \mathrm{H}) .{ }^{13} \mathrm{C}\left\{{ }^{1} \mathrm{H}\right\}$ NMR $(100 \mathrm{MHz}$, $\left.\mathrm{CDCl}_{3}\right): \delta 153.11,138.30,132.48,107.40,73.41,69.04,34.53,31.90,30.29,29.73$, $29.70,29.68,29.65,29.63,29.60,29.53,29.45,29.42,29.35,28.87,28.16,26.86,26.08$, $26.06,22.66,14.08$.

3,4,5-Trimethoxybenzyl bromide (7d). White solid (yield $=83 \%$ ). $\quad{ }^{1} \mathrm{H}$ NMR $\left(400 \mathrm{MHz}, \mathrm{CDCl}_{3}\right): \delta 6.62(\mathrm{~s}, 2 \mathrm{H}), 4.47(\mathrm{~s}, 2 \mathrm{H}), 3.87(\mathrm{~s}, 6 \mathrm{H}), 3.85(\mathrm{~s}, 3 \mathrm{H}) . \quad{ }^{13} \mathrm{C}\left\{{ }^{1} \mathrm{H}\right\}$ NMR (100 MHz, $\left.\mathrm{CDCl}_{3}\right): \delta 153.21,138.03,133.13,106.01,60.81,56.15,34.25$.

1,1'-Bis(3,4,5-trioctyloxybenzyl)-4,4'-bipyridinium bis(hexafluorophosphate) (1). A mixture of $7 \mathbf{a}(1.39 \mathrm{~g}, 2.5 \mathrm{mmol})$ and 4,4'-bipyridyl $(0.16 \mathrm{~g}, 1.0 \mathrm{mmol})$ in dry THF $(20 \mathrm{~mL})$ was stirred for $24 \mathrm{~h}$ at $40{ }^{\circ} \mathrm{C}$. The reaction mixture was concentrated under a reduced pressure. The residue was dissolved in methanol with heating. To this solution was added an aqueous solution $(10 \mathrm{~mL})$ of $\mathrm{NH}_{4} \mathrm{PF}_{6}(0.49 \mathrm{~g}, 3.0 \mathrm{mmol})$. The formed precipitate was separated by filtration, and washed with water and methanol. The product was purified by column chromatography (silica, ethyl acetate as an eluent) and dried under vacuum to afford 1 as a yellow waxy solid (yield $=0.40 \mathrm{~g}, 29 \%$ ). $\quad{ }^{1} \mathrm{H}$ $\operatorname{NMR}\left(400 \mathrm{MHz}, \mathrm{CDCl}_{3}\right): \delta 8.81(\mathrm{~d}, J=6.4 \mathrm{~Hz}, 4 \mathrm{H}), 8.14(\mathrm{~d}, J=6.4 \mathrm{~Hz}, 4 \mathrm{H}), 6.70(\mathrm{~s}$, 4H), $5.63(\mathrm{~s}, 4 \mathrm{H}), 3.99-3.92(\mathrm{~m}, 12 \mathrm{H}), 1.78-1.70(\mathrm{~m}, 12 \mathrm{H}), 1.50-1.20(\mathrm{~m}, 60 \mathrm{H}), 0.86(\mathrm{t}$, $J=6.8 \mathrm{~Hz}, 18 \mathrm{H}) .{ }^{13} \mathrm{C}\left\{{ }^{1} \mathrm{H}\right\} \mathrm{NMR}\left(100 \mathrm{MHz}, \mathrm{CDCl}_{3}\right): \delta 154.15,150.13,144.92$, $139.36,126.77,125.73,107.99,73.50,69.23,65.64,31.88,31.81,30.34,29.53,29.41$, 29.36, 29.28, 26.12, 26.06, 22.66, 22.64, 14.04. MS (MALDI): $\mathrm{m} / z$ 1106.29 (calcd $\left.\left[M-2 \mathrm{PF}_{6}\right]^{+}=1106.90\right)$. Anal. calcd for $\mathrm{C}_{72} \mathrm{H}_{118} \mathrm{~F}_{12} \mathrm{~N}_{2} \mathrm{O}_{6} \mathrm{P}_{2}: \mathrm{C}, 61.87 ; \mathrm{H}, 8.51 ; \mathrm{N}$, $2.00 \%$; found: C, $62.10 ; \mathrm{H}, 8.81 ; \mathrm{N}, 1.72 \%$.

phosphate) (2). This compound was prepared from $7 \mathbf{b}$ (4.34 g, $6.0 \mathrm{mmol})$, 
4,4'-bipyridyl (0.39 g, $2.5 \mathrm{mmol})$ and $\mathrm{NH}_{4} \mathrm{PF}_{6}(1.22 \mathrm{~g}, 7.5 \mathrm{mmol})$. The product was washed with ethyl acetate repeatedly, and dried under vacuum to give $\mathbf{2}$ as a yellow solid (yield $=0.70 \mathrm{~g}, 16 \%) . \quad{ }^{1} \mathrm{H}$ NMR $\left(400 \mathrm{MHz}, \mathrm{CDCl}_{3}\right): \delta 8.82(\mathrm{~d}, J=6.4 \mathrm{~Hz}, 4 \mathrm{H})$, $8.15(\mathrm{~d}, J=6.4 \mathrm{~Hz}, 4 \mathrm{H}), 6.70(\mathrm{~s}, 4 \mathrm{H}), 5.63(\mathrm{~s}, 4 \mathrm{H}), 3.97-3.92(\mathrm{~m}, 12 \mathrm{H}), 1.78-1.70(\mathrm{~m}$, 12H), $1.50-1.20(\mathrm{~m}, 108 \mathrm{H}), 0.87(\mathrm{t}, J=6.8 \mathrm{~Hz}, 18 \mathrm{H}) .{ }^{13} \mathrm{C}\left\{{ }^{1} \mathrm{H}\right\}$ NMR $(100 \mathrm{MHz}$, $\left.\mathrm{CDCl}_{3}\right): \delta 154.17,150.14,144.94,139.39,126.80,125.72,108.01,73.52,69.26,65.65$ $31.93,30.39,29.75,29.70,29.64,29.54,29.39,26.18,26.12,22.67,14.08$. MS $(\mathrm{MALDI}): m / z \quad 1443.16$ (calcd $\left.\left[M-2 \mathrm{PF}_{6}\right]^{+}=1443.27\right)$. Anal. calcd for $\mathrm{C}_{96} \mathrm{H}_{166} \mathrm{~F}_{12} \mathrm{~N}_{2} \mathrm{O}_{6} \mathrm{P}_{2}: \mathrm{C}, 66.48 ; \mathrm{H}, 9.65 ; \mathrm{N}, 1.62 \%$; found: $\mathrm{C}, 66.33 ; \mathrm{H}, 9.85 ; \mathrm{N}, 1.61 \%$.

1,1'-Bis(3,4,5-trihexadecyloxybenzyl)-4,4'-bipyridinium bis(hexafluorophosphate) (3). This compound was prepared from 7c (2.14 g, $2.4 \mathrm{mmol})$, 4,4'-bipyridyl $(0.16 \mathrm{~g}, 1.0 \mathrm{mmol})$ and $\mathrm{NH}_{4} \mathrm{PF}_{6}(0.49 \mathrm{~g}, 3.0 \mathrm{mmol})$. The product was washed with ethyl acetate repeatedly, and dried under vacuum to give $\mathbf{3}$ as a yellow

solid (yield $=0.53 \mathrm{~g}, 26 \%) . \quad{ }^{1} \mathrm{H}$ NMR $\left(400 \mathrm{MHz}, \mathrm{CDCl}_{3}\right): \delta 8.84(\mathrm{~d}, J=6.4 \mathrm{~Hz}, 4 \mathrm{H})$, $8.16(\mathrm{~d}, J=6.4 \mathrm{~Hz}, 4 \mathrm{H}), 6.71(\mathrm{~s}, 4 \mathrm{H}), 5.64(\mathrm{~s}, 4 \mathrm{H}), 3.97-3.92(\mathrm{~m}, 12 \mathrm{H}), 1.78-1.70(\mathrm{~m}$, 12H), $1.50-1.20(\mathrm{~m}, 156 \mathrm{H}), 0.87(\mathrm{t}, J=6.8 \mathrm{~Hz}, 18 \mathrm{H}) .{ }^{13} \mathrm{C}\left\{{ }^{1} \mathrm{H}\right\}$ NMR $(100 \mathrm{MHz}$, $\left.\mathrm{CDCl}_{3}\right): \delta 154.22,150.19,144.94,139.47,126.84,125.62,108.04,73.54,69.29,65.72$, $31.96,31.43,30.01,29.78,29.74,29.71,29.60,29.45,29.41,26.22,26.16,22.71,14.12$. MS (MALDI): $m / z$ 1779.62 (calcd $\left[M-2 \mathrm{PF}_{6}\right]^{+}=$1779.65). Anal. calcd for $\mathrm{C}_{120} \mathrm{H}_{214} \mathrm{~F}_{12} \mathrm{~N}_{2} \mathrm{O}_{6} \mathrm{P}_{2}: \mathrm{C}, 69.60 ; \mathrm{H}, 10.42 ; \mathrm{N}, 1.35 \%$; found: 69.45; H, 10.66; N, 1.33\%.

\section{1,1'-Bis(3,4,5-trimethoxybenzyl)-4,4'-bipyridinium bis(hexafluorophosphate)}

(4). A mixture of 7d (2.61 g, $10 \mathrm{mmol})$ and 4,4'-bipyridyl (0.63 g, $4.0 \mathrm{mmol})$ in dry THF $(15 \mathrm{~mL})$ was stirred for $12 \mathrm{~h}$ at $40{ }^{\circ} \mathrm{C}$. A solution of $\mathrm{NH}_{4} \mathrm{PF}_{6}(1.63 \mathrm{~g}, 10 \mathrm{mmol})$ in acetonitrile $(10 \mathrm{~mL})$ was slowly added to the reaction mixture to form a yellow precipitate. The formed precipitate was collected by filtration. The product was 
washed with water, methanol and hexane, successively, and dried under vacuum to afford 4 as a yellow solid (yield $=3.08 \mathrm{~g}, 95 \%) . \quad{ }^{1} \mathrm{H}$ NMR (400 MHz, DMSO- $\left.d_{6}\right)$ : $\delta 9.49(\mathrm{~d}, J=6.8 \mathrm{~Hz}, 4 \mathrm{H}), 8.68(\mathrm{~d}, J=6.8 \mathrm{~Hz}, 4 \mathrm{H}), 7.04(\mathrm{~s}, 4 \mathrm{H}), 5.77(\mathrm{~s}, 4 \mathrm{H}), 3.79(\mathrm{~s}$, 12H), $3.63(\mathrm{~s}, 6 \mathrm{H}) .{ }^{13} \mathrm{C}\left\{{ }^{1} \mathrm{H}\right\}$ NMR (100 MHz, DMSO- $\left.d_{6}\right): \delta 153.39,149.24,145.47$, 138.38, 129.10, 127.11, 107.03, 63.95, 60.03, 56.12. MS (MALDI): $\mathrm{m} / z 518.31$ (calcd $\left.\left[M-2 \mathrm{PF}_{6}\right]^{+}=518.24\right)$
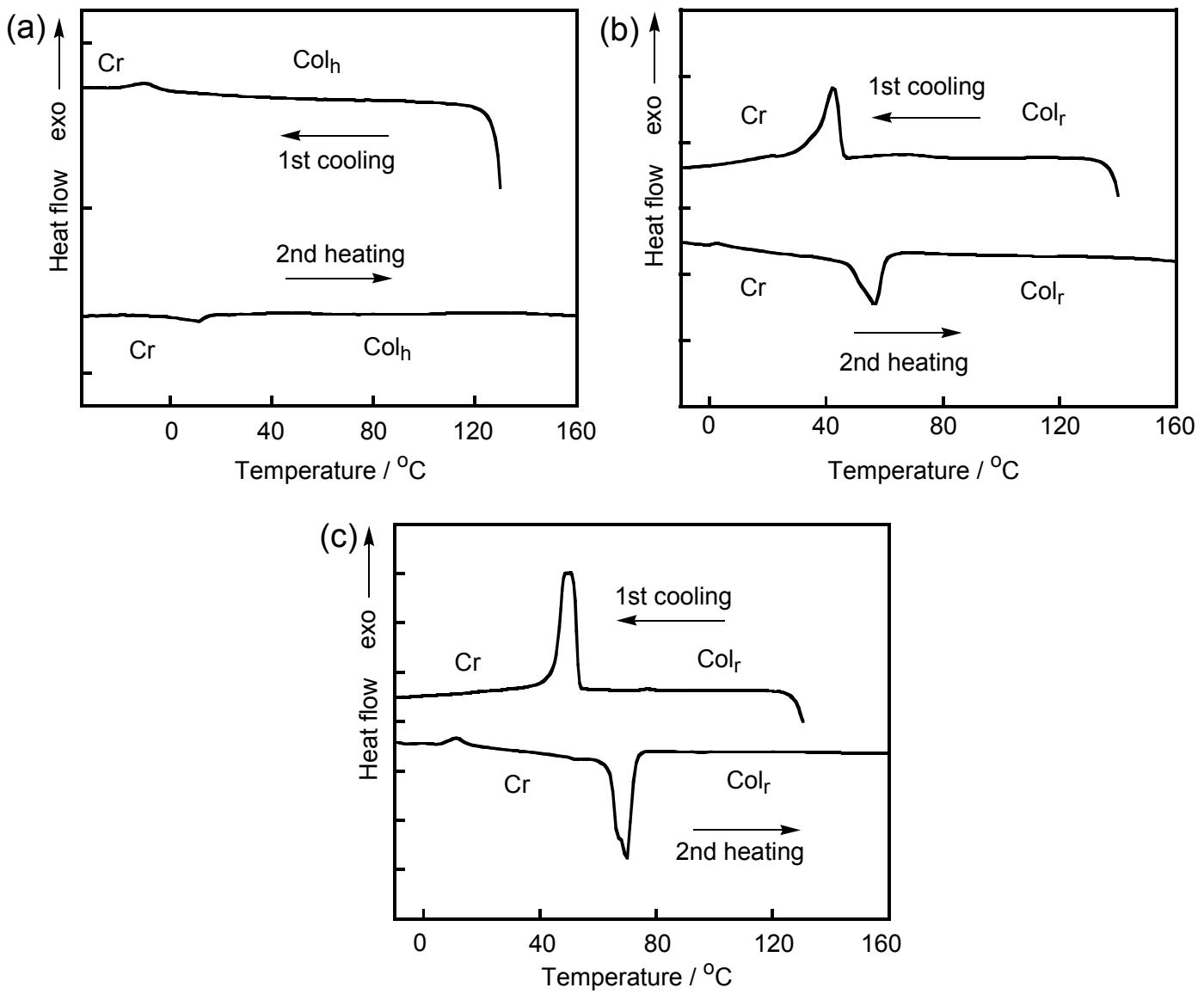

Figure S1. DSC thermograms of (a) 1, (b) 2, and (c) 3 at a scanning rate of $10^{\circ} \mathrm{C}$ $\min ^{-1}$. Cr: crystalline; $\mathrm{Col}_{\mathrm{h}}$ : hexagonal columnar; $\mathrm{Col}_{\mathrm{r}}$ : rectangular columnar. 


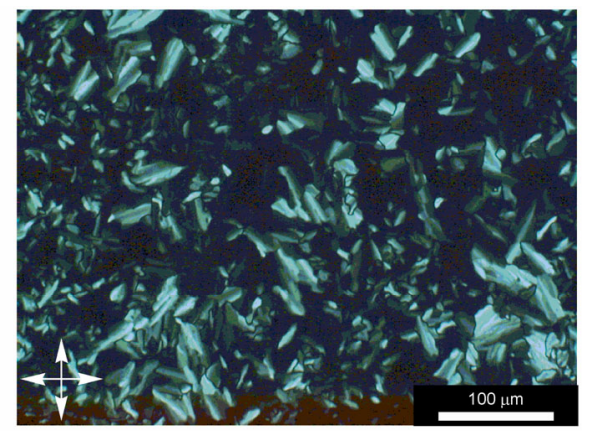

Figure S2. Polarized optical photomicrograph of $\mathbf{1}$ in the $\mathrm{Col}_{\mathrm{h}}$ phase at $100{ }^{\circ} \mathrm{C}$.

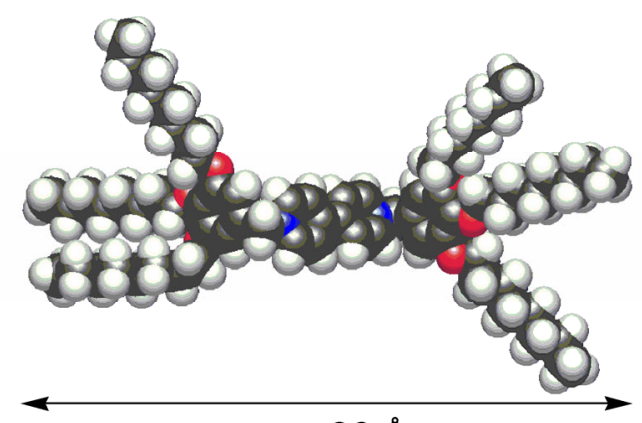

ca. $36 \AA$

Figure S3. Energy minimized molecular structure of $\mathbf{1}$.

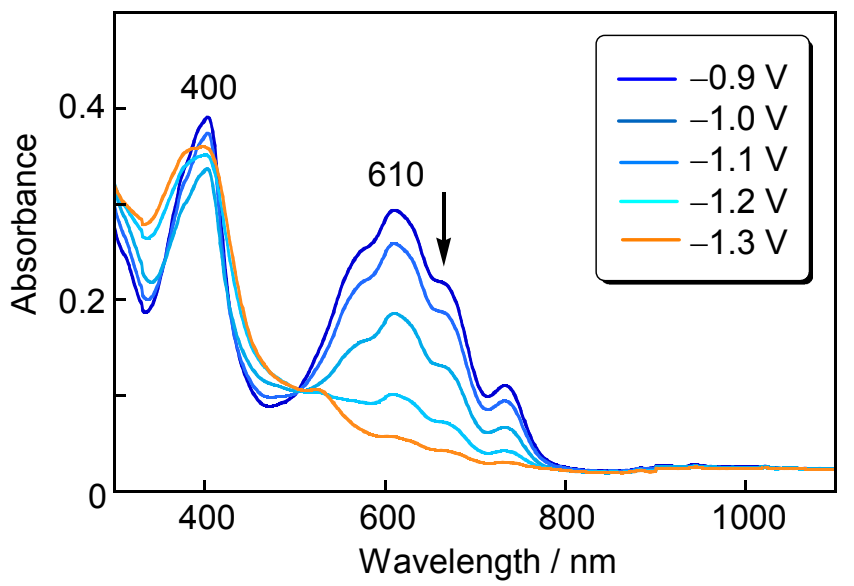

Figure S4. UV-vis-NIR spectra of viologen $1(1.0 \mathrm{mM})$ observed during the second reduction step in a $\mathrm{CH}_{2} \mathrm{Cl}_{2}$ solution of $\mathrm{Bu}_{4} \mathrm{NClO}_{4}(0.10 \mathrm{M})$ using a $\mathrm{Pt}$ working electrode. An arrow indicates the direction of spectral changes. 


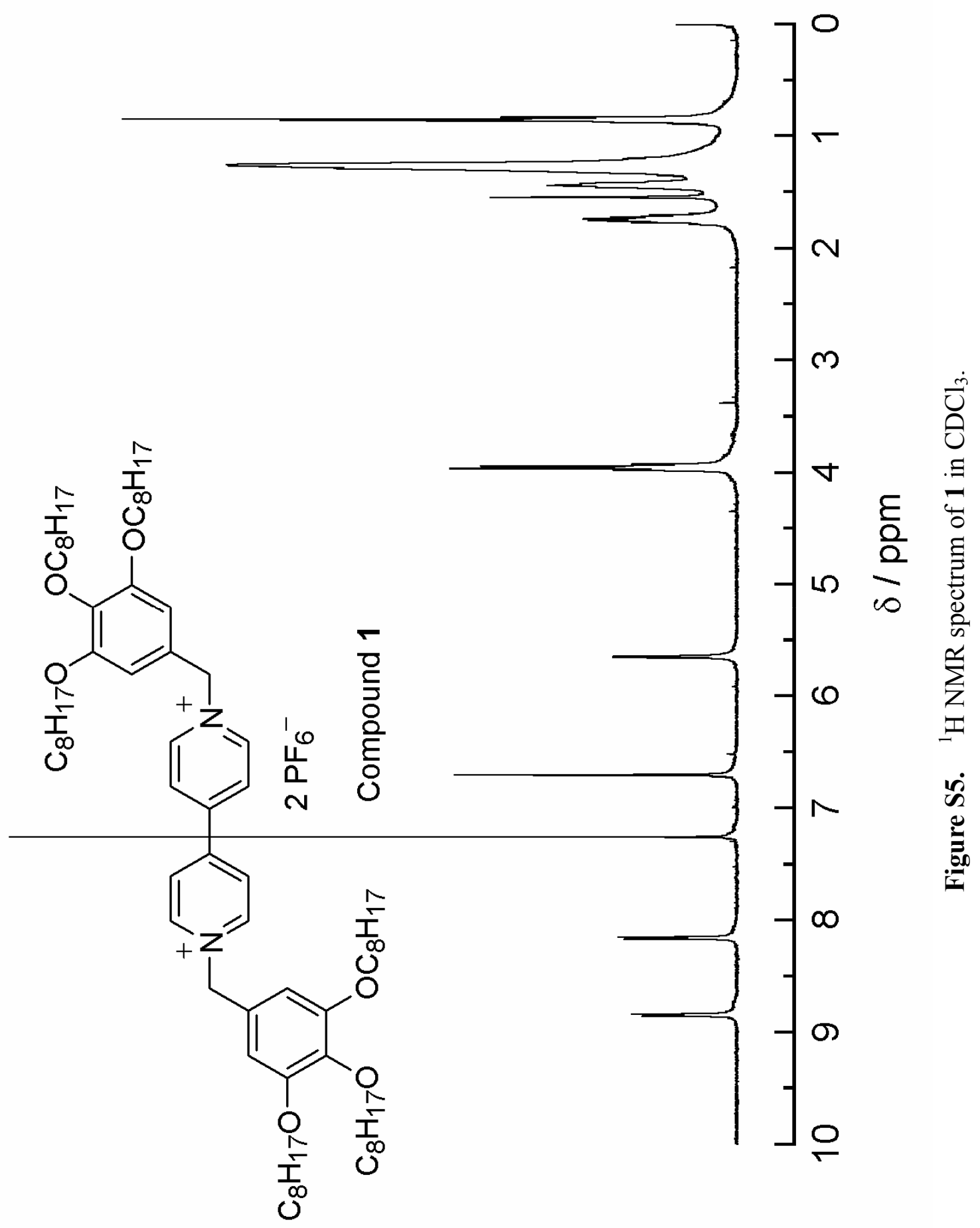




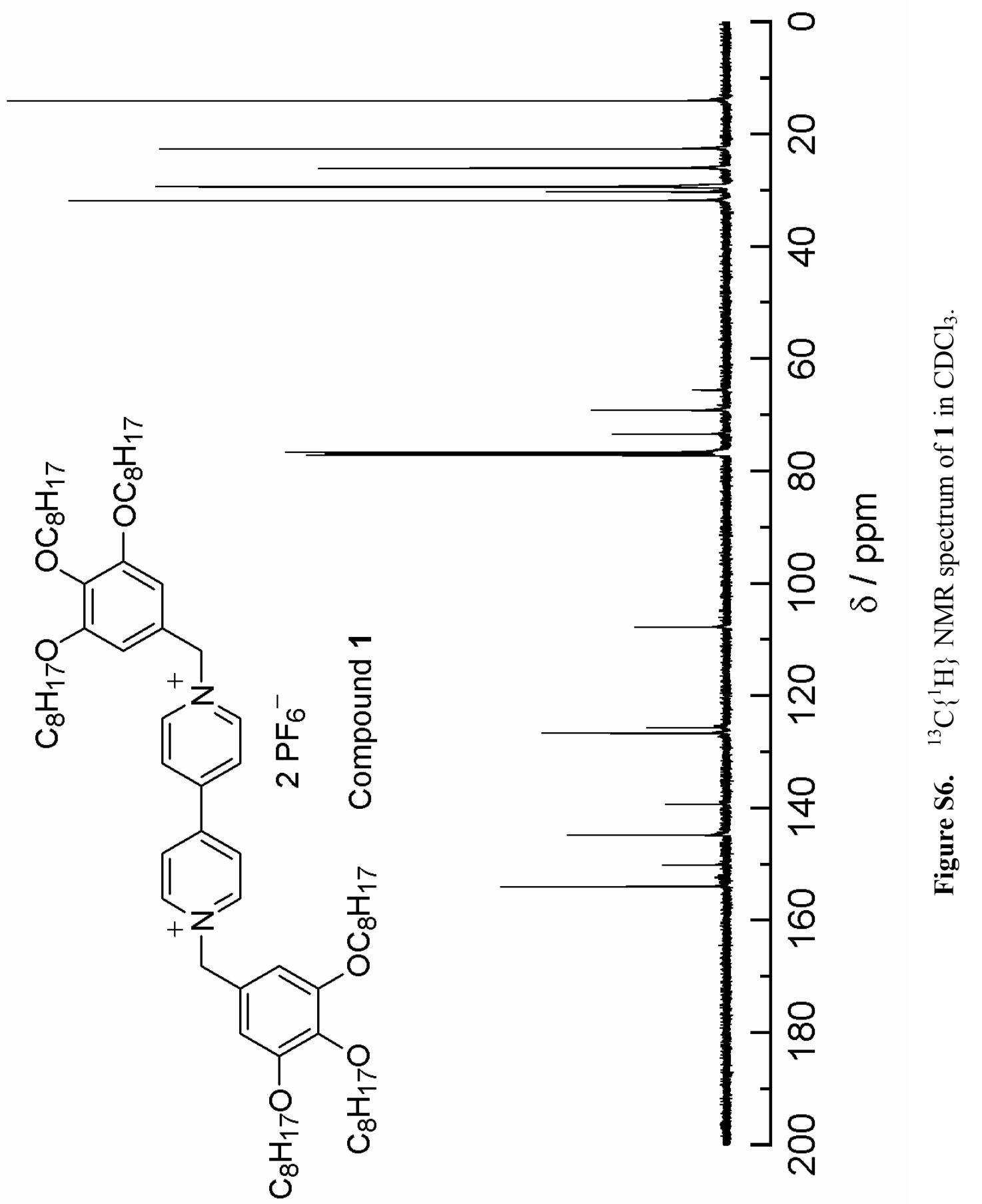




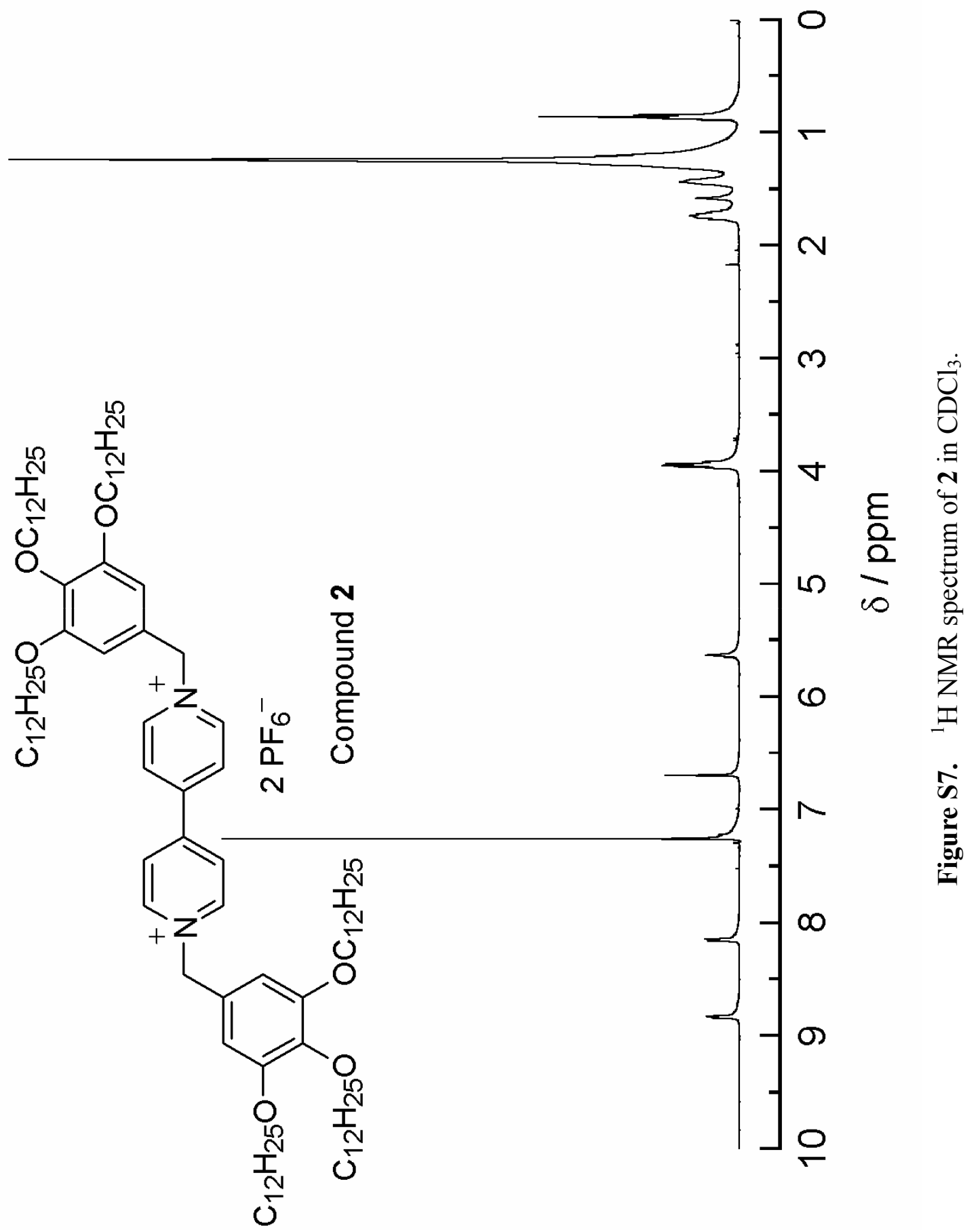




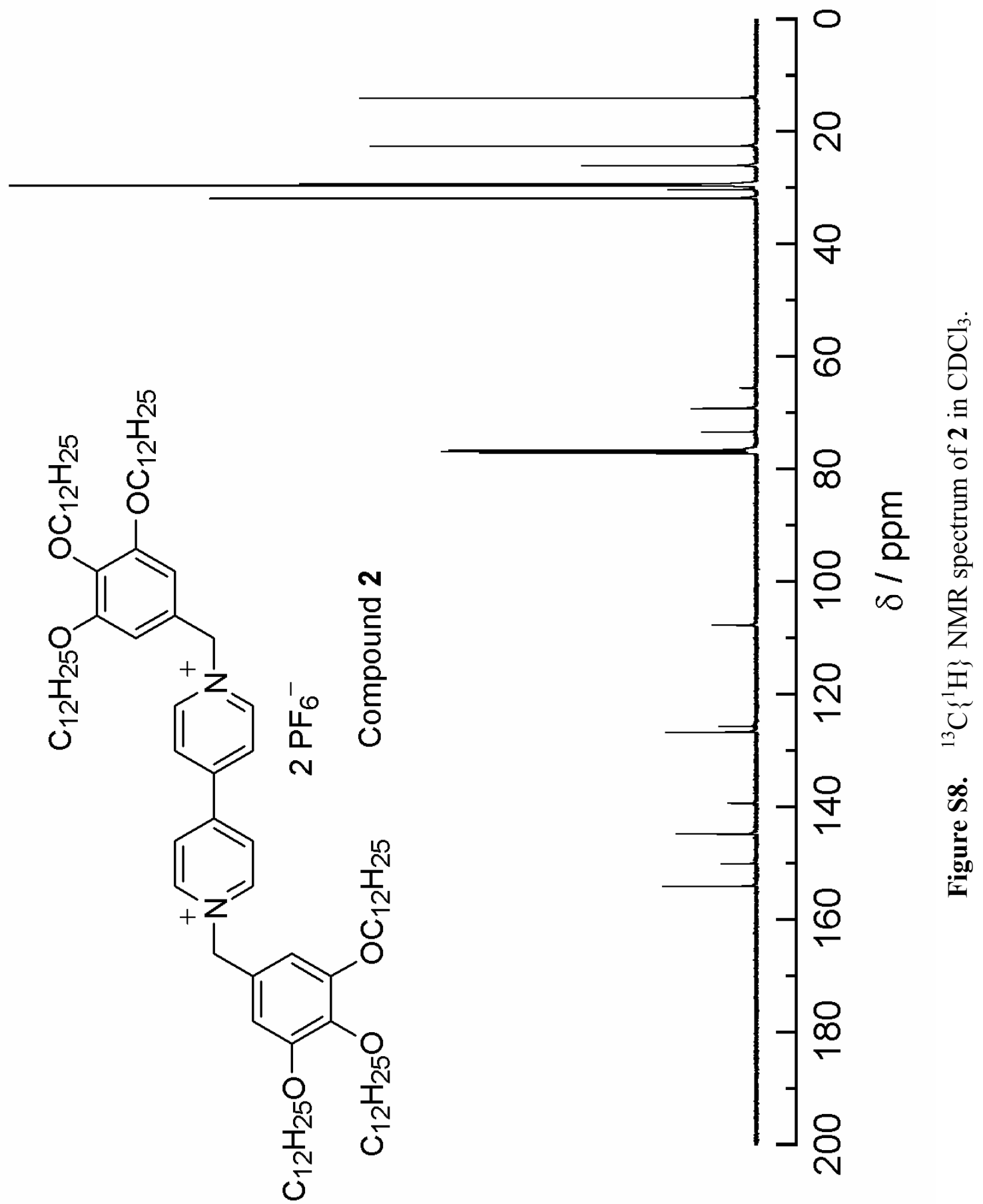




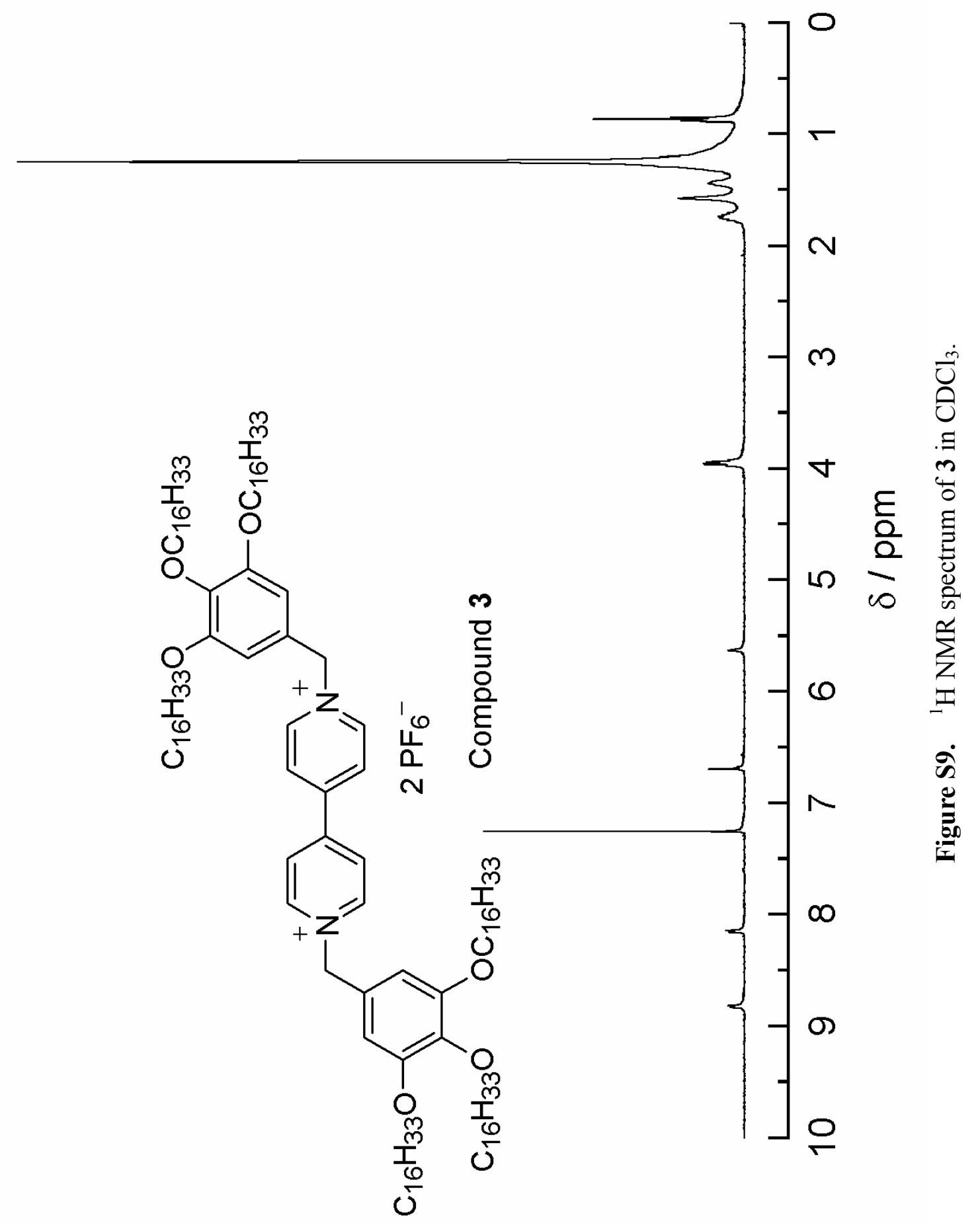




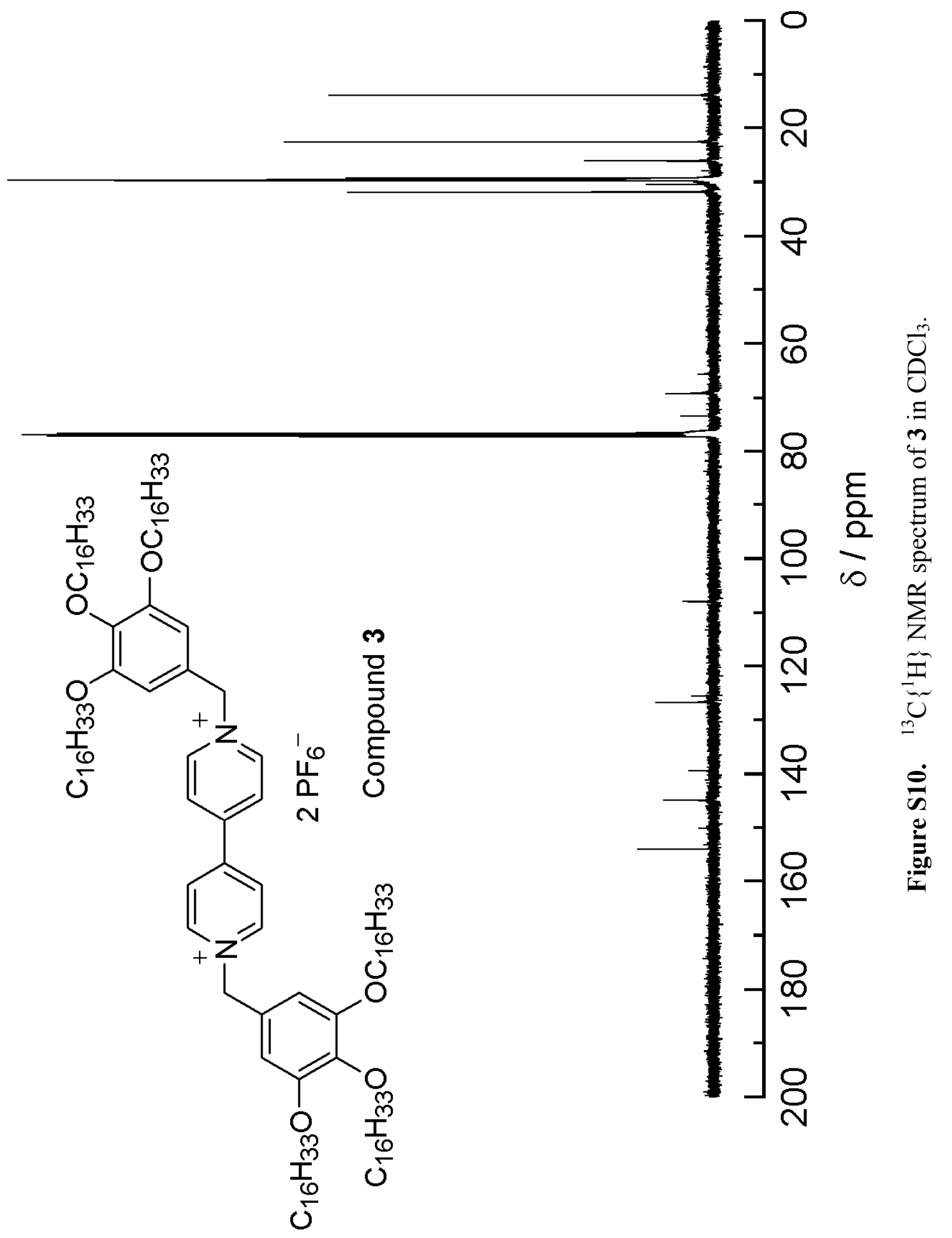




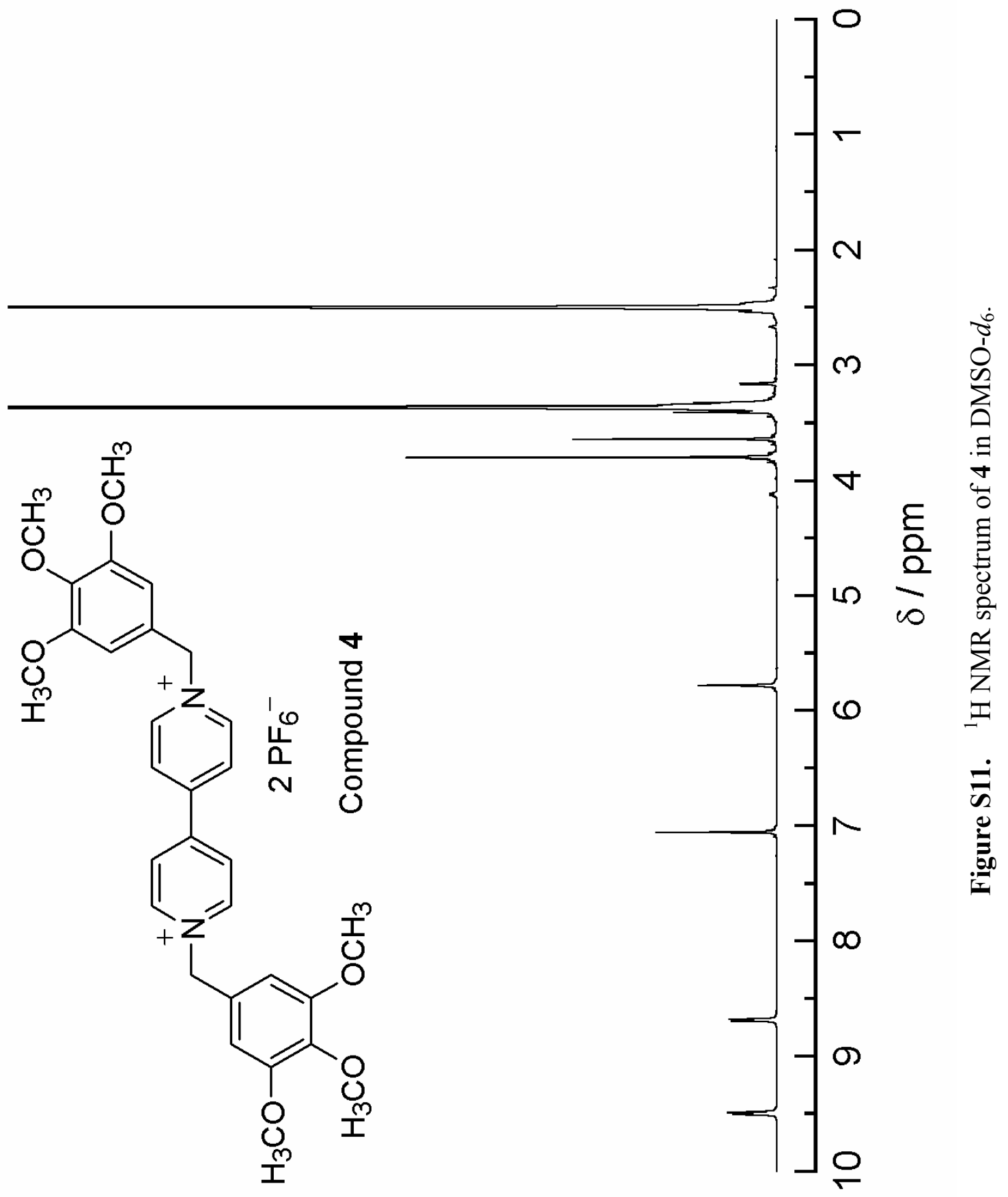




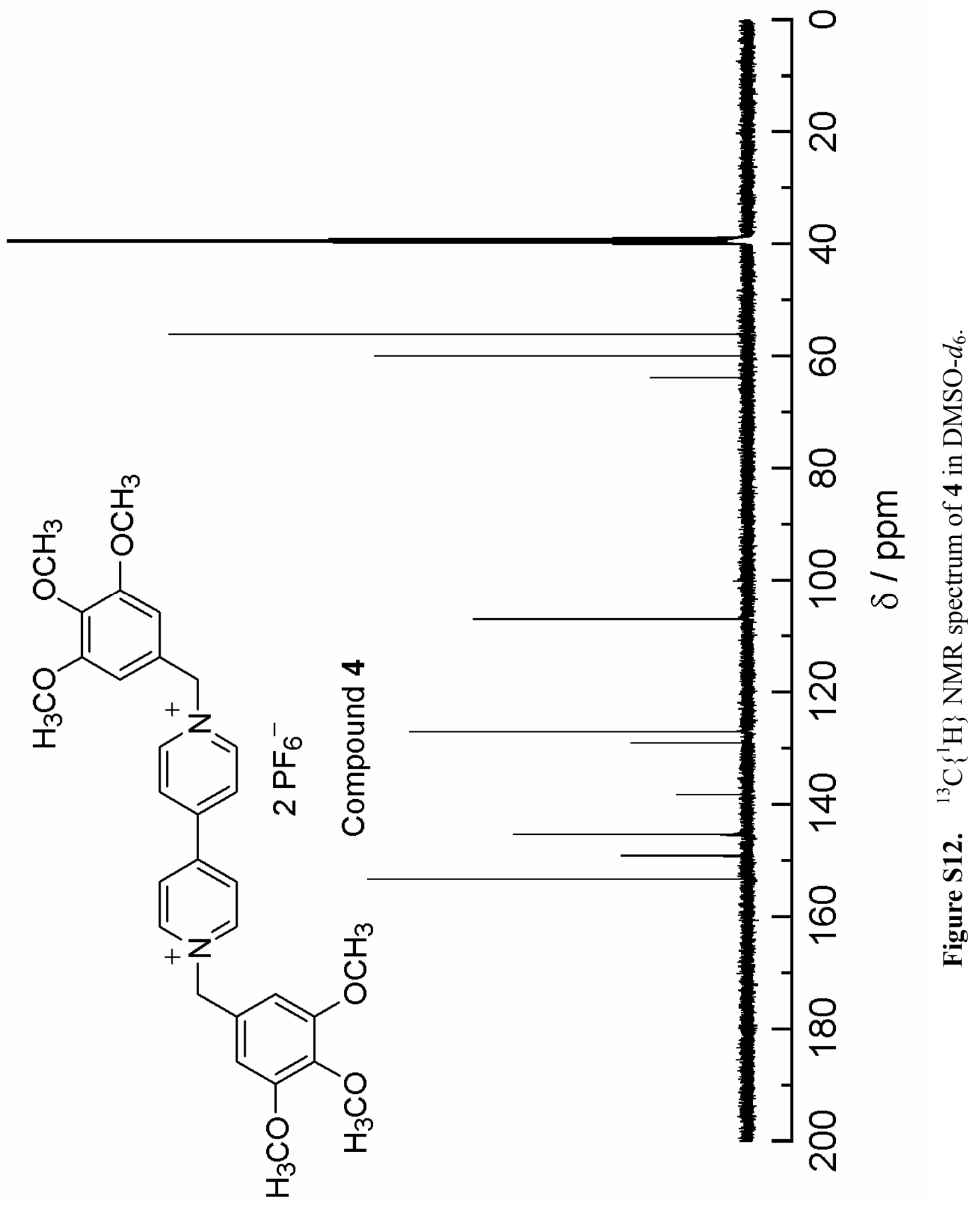



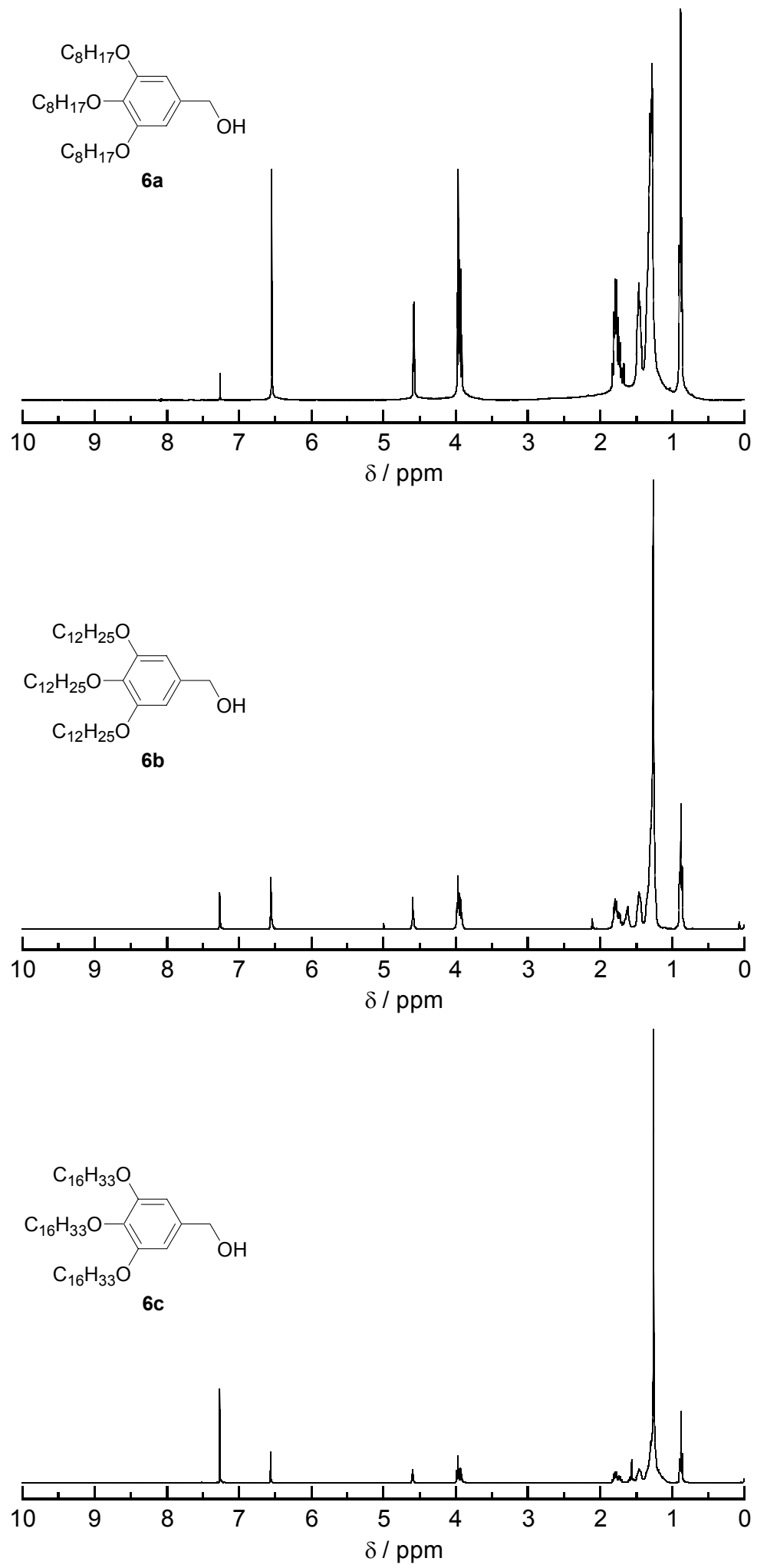

Figure S13. $\quad{ }^{1} \mathrm{H}$ NMR spectra of 6a-c in $\mathrm{CDCl}_{3}$. 

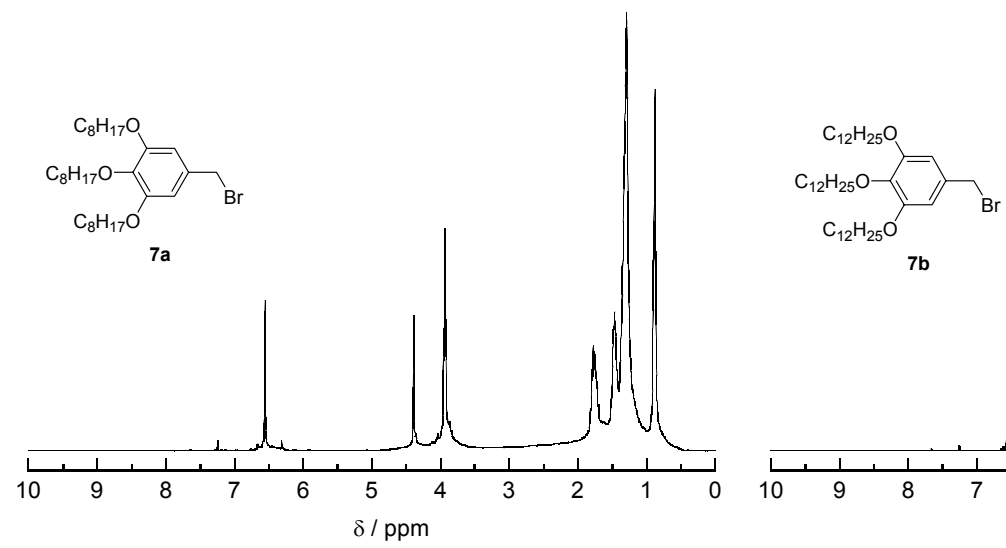

$7 b$

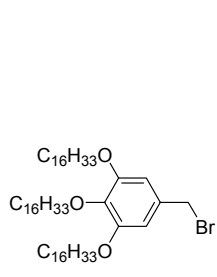

$7 \mathrm{c}$
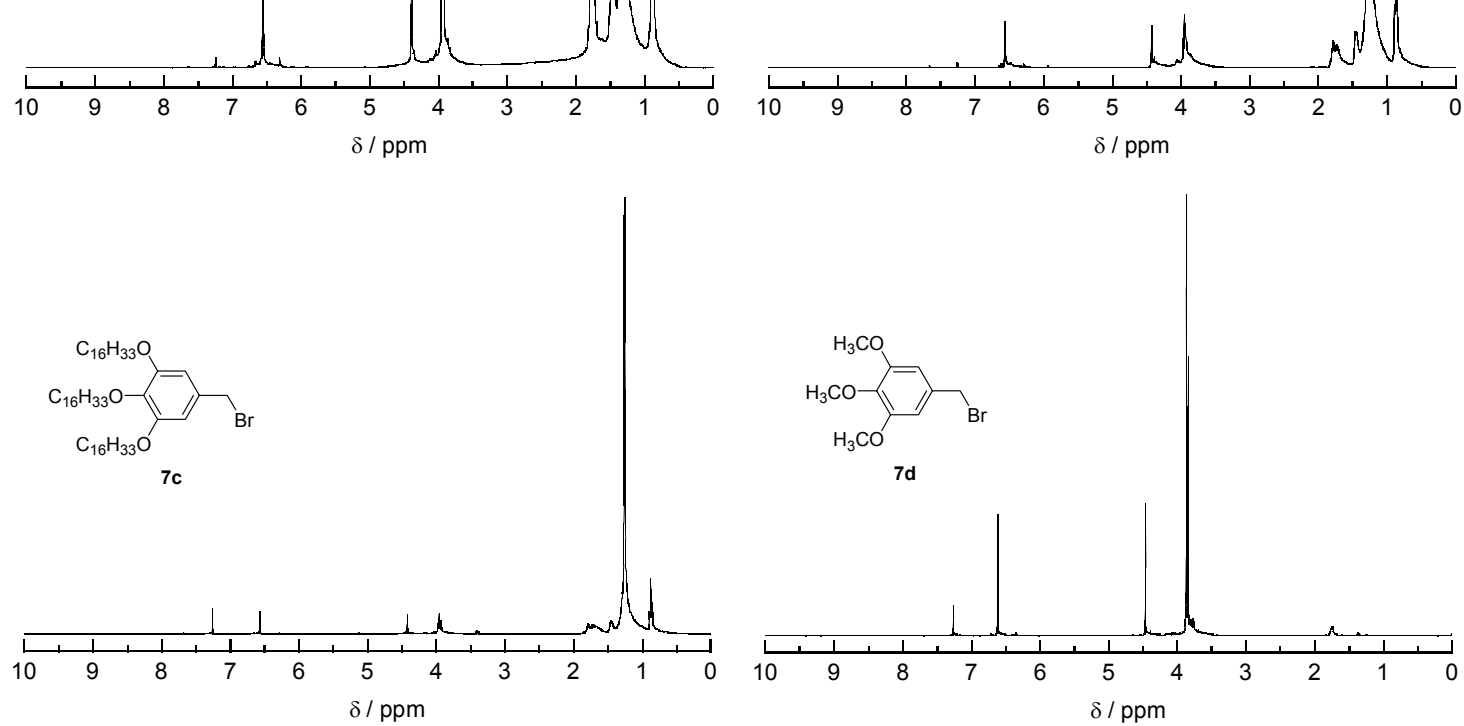

Figure S14. $\quad{ }^{1} \mathrm{H}$ NMR spectra of $\mathbf{7 a - d}$ in $\mathrm{CDCl}_{3}$.

\section{References.}

(1) Altomare, A.; Burla, M. C.; Camalli, M.; Cascarano, G. L.; Giacovazzo, C.; Guagliardi, A.; Moliterni, A. G. G.; Polidori, G.; Spagna, R. J. Appl. Crystallogr. 1999, $32,115$.

(2) Sheldrick, G. M. SHELXL-97, Program for the Refinement of Crystal Structures; University of Göttingen: Göttingen, Germany, 1997.

(3) (a) Percec, V.; Schlueter, D.; Ronda, J. C.; Johansson, G.; Unger, G.; Zhou, J. P. Macromolecules 1996, 29, 1464 . (b) Serrette, A. G.; Lai, C. K.; Swager, T. M. Chem. Mater. 1994, 6, 2252. (c) Lai, C. K.; Tsai, C.-H.; Pang, Y.-S. J. Mater. Chem. 1998, 8, 1355. (d) Cheng, F.; Adronov, A. Chem. Eur. J. 2006, 12, 5053. (e) Yoshio, M.; Mukai, T.; Ohno, H.; Kato, T. J. Am. Chem. Soc. 2004, 126, 994. 